\title{
Quasi-Single Mode Operated Few-Mode Fiber for Distributed
} Acoustic Sensing

\author{
Islam Ashry, ${ }^{\mathrm{a}, \dagger}$ Yuan Mao, ${ }^{\mathrm{a}, \dagger}$ Tien Khee Ng, ${ }^{\mathrm{a}}$ Frode Hveding, ${ }^{\mathrm{b}, \#}$ Muhammad Arsalan, ${ }^{\mathrm{b}}$ and Boon S. \\ $\mathrm{Ooi}^{\mathrm{a}, *}$ \\ ${ }^{a}$ King Abdullah University of Science and Technology (KAUST), Computer, Electrical, and \\ Mathematical Sciences and Engineering, Thuwal 23955-6900, Saudi Arabia \\ ${ }^{b}$ EXPEC Advanced Research Center, Saudi Aramco, Dhahran 34464, Saudi Arabia \\ ${ }^{\dagger}$ These authors contributed equally to this work \\ "\#frode.hveding@aramco.com, *boon.ooi@kaust.edu.sa
}

\begin{abstract}
We experimentally report a proof-of-concept demonstration of a few-mode fiber (FMF) based distributed acoustic sensor (DAS) design, aiming at upgrading the capabilities of the typical DAS that employs the standard single mode fiber (SMF). We only excite the fundamental mode at the input port of the FMF, and further, we minimize the impact of intermodal coupling within it such that the FMF operates in a quasi-single mode (QSM) state. The QSM operated FMF keeps the basic operation principle of the DAS valid and, in comparison with the standard SMF, it allows injection of higher pump peak-power before reaching the threshold power of nonlinearity. We validate our design by sensing vibration events produced by a piezoelectric transducer (PZT) cylinder. The FMF based DAS successfully figures out the locations and frequencies of these events. This reported design would enable the realization of a DAS design with longer sensing range and higher spatial resolution, in comparison to the standard SMF based DAS.
\end{abstract}

Keywords: Few-mode fiber, Acoustic sensors, optical fiber sensors, optical fibers, optical sensors.

\section{INTRODUCTION}

The recent research on few-mode fibers (FMFs) has developed numerous important applications, including optical communications and optical sensing [1,2]. In particular, space-division-multiplexing in a FMF-based communication network can significantly increase its overall data transfer rate via using each linearly-polarized (LP) mode as a separate communication channel. FMFs have been also deployed to improve the capabilities of optical sensing. Since each LP mode in a FMF interacts differently with the sensing parameters, FMF-based sensors can monitor multiple parameters simultaneously. For example, writing FBGs in FMFs has been used to simultaneously sense temperature and strain [3], and bending and strain [4]. Other trials show that FMFs can construct large scale absorption-based sensing network [5] and reconstruct spatial distributions of gases that diffuse into a silica FMF [6], functionalities that are difficult to implement using the standard single-mode fiber (SMF) and multimode fiber (MMF).

Because distributed optical fiber sensors have been widely deployed in many important applications and given the unique specifications of FMFs, investigating the behavior of such sensors with FMFs has recently attracted considerable research interest. For instance, a two-mode fiber (TMF)-based Brillouin optical time-domain reflectometry (BOTDR) can offer simultaneous distributed strain and temperature sensing [7]. Another trials in Ref. [8] shows a TMF can extend the sensing range of the typical MMF-based distributed temperature sensor (DTS). Although the Rayleigh distributed acoustic sensor (DAS) finds many substantial applications, its behavior when using a FMF has not been widely explored $[9,10]$.

Here, we report a proof-of-concept demonstration of designing DAS using a FMF. In this design, we excite only the fundamental mode (i.e., the $\mathrm{LP}_{01}$ mode) in the FMF such that it operates in the quasi-single mode (QSM) state. We prove that with careful selection of the technique of injecting light into the FMF and by utilizing proper FMF type, intermodal coupling between the $\mathrm{LP}_{01}$ mode and the other higher order modes can be largely suppressed. The performance of the designed FMF based DAS is examined through detecting different vibration events produced by a piezoelectric transducer (PZT) cylinder. We find that for these different events, vibrations can be accurately sensed with high SNR. Additionally, we compare the performances of the FMF and standard SMF based DAS to find that both possess 
comparable SNR. One pivotal advantage of our system is that the QSM operation in the FMF offers larger effective area to the fundamental mode (i.e., higher threshold power of nonlinearity), in comparison with the standard SMF, and simultaneously does not violate the requirements of the operation principle of DAS system (i.e., presence of singular mode in the fiber). Furthermore, the QSM operation can alleviate the spatial resolution deterioration caused by modal dispersion in fibers that supports propagating multiple modes. Consequently, the work presented here can serve as the first step towards upgrading conventional DAS system, in terms of sensing range and spatial resolution, and also integrating DAS with the other optical fiber distributed sensing techniques that utilize MMF.

\section{DAS OPERATION IN THE QSM STATE}

The basic operation of the optical fiber DAS consists of launching a train of optical pulses, generated by a narrow linewidth laser, into a fiber under test (FUT). At the FUT input port, consecutive Rayleigh backscattered traces are recorded in the time domain. Each Rayleigh trace has a speckle-like profile because of coherent interference of the signals reflected by scattering centers within the injected pulse duration $\left[{ }^{11}\right]$. In the absence of intrusion along the FUT, i.e., no RI perturbation within the FUT, the recorded Rayleigh traces are ideally identical. In case of an acoustic event is applied at a position along the FUT, the effective RI changes at this position and consequently, the intrusion could be sensed by observing the intensity fluctuation of its corresponding speckle in the recorded traces. Fundamentally, if the FUT supports propagating a large number of modes, similar to the case of MMF, the SNR of the DAS system would be significantly degraded. This is because the backscattered Rayleigh signal of any section along the fiber becomes the resultant of the interference behaviors of the individual modes. Thus, on average with a large number of modes, the Rayleigh signal would not be sensitive to the vibration event.

In our experiments, the used FUT is a step-index fiber (YOFC Co., Ltd.) of V-number equals 3.27 at $1535 \mathrm{~nm}$ operation wavelength. Thus, the fiber is a TMF that supports propagating the $\mathrm{LP}_{01}$ and $\mathrm{LP}_{11}$ mode. To satisfy the operation requirements of $\Phi$-OTDR based DAS, one should selectively excite a single spatial mode at the TMF input port and this mode keeps propagating along the fiber with minimal intermodal coupling. One major advantage of the step-index profile of the TMF is its ability to significantly suppress intermodal coupling between the two spatial modes $\left[{ }^{12}\right]$. To provide feasibility to our design, we select the QSM operation for the TMF by injecting only the LP $\mathrm{P}_{01}$ mode into the TMF. We start our analysis with a control experiment using the setup in Fig. 1(a) to prove the capability of the QSM operation in the TMF even when introducing RI perturbations, similar to those sensed by $\Phi$-OTDR based DAS, along the TMF. As shown in Fig. 1(a), we use a single mode pigtail fiber coupled laser that generates continuous wave (CW) light at $1535 \mathrm{~nm}$ wavelength. Efficient transfer of the $\mathrm{LP}_{01}$ mode from the SMF to the TMF can be performed via aligning their axes followed by joining them through either fusion splicing or mating sleeve. Microscope image of the two fibers after fusion splicing and schematic for their connection with the mating sleeve are shown in the inset. 1 and inset. 2 of Fig. 1(a), respectively. The length of the TMF is $\sim 3.1 \mathrm{~km}$ and we attach a $10 \mathrm{~m}$ section of it, near its end, to a PZT cylinder such that its vibration frequency is controllable via a function generator. PZT cylinder is the typical vibration source used for the calibration of $\Phi$-OTDR based DAS $\left[{ }^{13}\right]$. In this control experiment, however, we use the PZT for another purpose which is investigating the impact of the RI change induced by the PZT on the intermodal

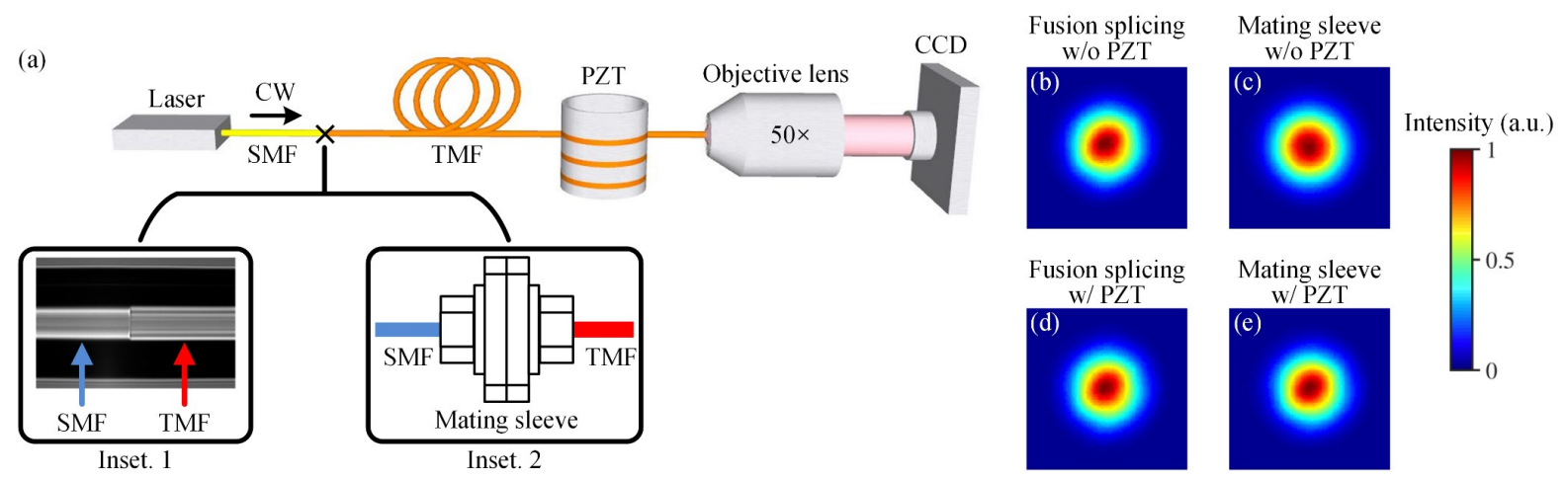

Figure. 1. (a) Experimental setup for verifying the QSM operation within the TMF; SMF-TMF joining with fusion splicing (Inset. 1) or mating sleeve (Inset. 2). Intensity profiles at the TMF output port when the TMF is joined to the SMF with (b) fusion splicing and (c) mating sleeve while the PZT is static, and with (d) fusion splicing and (e) mating sleeve while the PZT is vibrating with $5 \mathrm{kHz}$ frequency. 
coupling within the TMF. At the output end of the fiber, an objective lens $(50 \times, \mathrm{NA}=0.45)$ and a near-infrared CCD camera (Ophir Optronics, SP503U-1550) are used to image the TMF output intensity profile. The output intensity profiles when joining the SMF and TMF using fusion splicing and mating sleeve are shown in Figs. 1(b) and 1(c), correspondingly. The results of these figures indicate that the fundamental mode is well excited at the TMF input port and maintains its profile, with almost negligible crosstalk with the $\mathrm{LP}_{11}$ mode, until reaching the TMF end. Next, we repeat imaging the output beam profiles for the SMF-TMF connection with fusion splicing/mating sleeve when the PZT is vibrating with a $5 \mathrm{kHz}$ arbitrary frequency, as shown in Fig. 1(d)/Fig. 1(e). Again, the $\mathrm{LP}_{01}$ mode preserves its shape at the TMF end which indicates the two modes are well spatially separated, even with introducing RI change within the TMF using the PZT. As a result, we prove the QSM operation within the TMF is feasible provided that selecting the proper fiber RI profile and the mode excitation method.

\section{SENSING THE PZT VIBRATION EVENTS}

In this section, we test the performance of the FMF based DAS in terms of discovering a location along the fiber subjected to a vibration event and calculating the frequency components of this vibration. We select the vibration sources to be a PZT cylinder because it is traditionally used to calibrate DAS since its vibration frequency can be predetermined using its driving function generator. The experimental setup of the $\Phi$-OTDR based DAS using the TMF is shown in Fig. 2. Narrow linewidth laser source (NKT Photonics, 15E) generates CW light of $16 \mathrm{dBm}$ optical power, 100 $\mathrm{Hz}$ linewidth, and $1535 \mathrm{~nm}$ operation wavelength. The laser light is modulated by an electro-optic modulator (EOM1) (Covega, Mach-40 085) to produce optical pulses with $20 \mathrm{kHz}$ repetition rate and $100 \mathrm{~ns}$ width which offers $10 \mathrm{~m}$ sensing spatial resolution. Since the EOM1 is sensitive to the input light polarization state, we use a polarization controller (PC1) to maximize the peak output power of the generated pulses by EOM1. To enhance the extinction ratio (ER) of the optical pulses, another electro-optic modulator (EOM2) (Covega, Mach-10 063) is synchronized with the EOM1 through a pulse generator $\left.{ }^{14}\right]$. Again, a polarization controller (PC2) tunes the peak-power of the pulses produced by EOM2 to its maximum value. The modulated light is then amplified by an erbium-doped-fiber-amplifier (EDFA1) to be launched through a circulator into the TMF, characterized in the previous section, of $\sim 3.1 \mathrm{~km}$ length. The backscattered Rayleigh traces from the TMF are recorded through direct detection. The traces are firstly amplified by another erbium-doped-fiber-amplifier (EDFA2) whose amplified spontaneous emission (ASE) noise is filtered out by a fiber Bragg grating (FBG). Finally, the backscattered traces are detected by a photodetector (PD) (Thorlabs, PDB 470C) and sampled at $125 \mathrm{MHz}$ rate using an oscilloscope (Tektronix, DPO70404C).

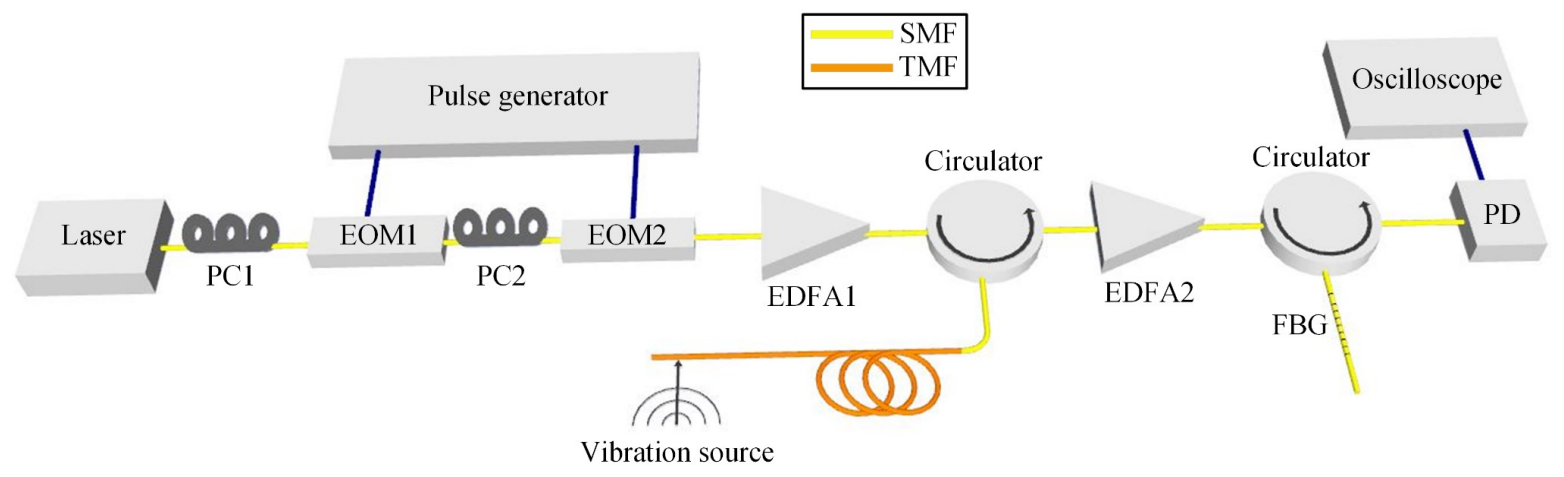

Figure. 2. Experimental schematic of the $\Phi-O T D R$ based DAS using a TMF.

We find that the DAS performance is almost the same when joining the SMF and TMF with fusion splicing or mating sleeve. Therefore, in the subsequent analysis, we only present the results of the case for mating sleeve. To figure out a position along the fiber subjected to a vibration signal, the recorded raw Rayleigh traces are first averaged, and then the consecutive averaged traces are subtracted. Assuming each averaged trace is calculated using $N$ consecutive traces, the effective sampling rate reduces to equal the repetition rate of injecting pulses divided by $N$. As a result, based on the sampling theory, the maximum detectable frequency (Nyquist frequency) is shrunk down by a factor of $N$, in comparison with the no averaging case. Based on our experimental parameters, in case we do not apply traces averaging, the maximum sensed vibration frequency equals $10 \mathrm{kHz}$. In our analysis, we do not use a fixed $N$ value. Instead, we divide the $10 \mathrm{kHz}$ range into 10 spectrum windows such that the $i$ th window starts at zero frequency and ends at $i \mathrm{kHz}$, where $i \in[1,2, \ldots, 10]$. For the $i$ th frequency window, we set $N=\lfloor 10 / i\rfloor$. The recorded Rayleigh traces are analyzed in parallel 
through the individual windows. After subtracting the averaged Rayleigh traces in each window, the vibration location can be identified in some windows, based on the vibration frequency. Then, for each identified vibration location, the vibration frequency can be calculated by applying the fast Fourier transform to the averaged Rayleigh traces at this position. Finally, we select the analysis of the window that covers all the frequency components of the vibration event and has the larger $N$ value. This is because typically the larger the $N$ value, the higher the SNR. This technique fits the real practical applications where the vibration frequency is unknown.

We start with detecting PZT vibrations by attaching a $10 \mathrm{~m}$ section of the TMF near its end to the PZT. As a representative example, Fig. 3(a) shows 100 consecutive averaged Rayleigh traces, where $N=10$, recorded when the PZT vibrates sinusoidally with $200 \mathrm{~Hz}$ frequency. The zoom-in image of the Rayleigh traces around the PZT location clearly shows their temporal power variations [inset of Fig. 3(a)]. In Fig. 3(b), we show the vibrations location information calculated within $45 \mathrm{~ms}$ through subtracting the traces presented in Fig. 3(a). Clearly, the vibration position can be determined. Figure 3(c) is the corresponding power spectrum of the $200 \mathrm{~Hz}$ vibrations event which exhibits accurate frequency detection. The appearance of high-order harmonics in the vibration power spectrum is due to the nonlinearity of the direct detection system. We further provide another example when driving the PZT with $10 \mathrm{kHz}$ vibration frequency, the maximum detectable vibration frequency by our experimental setup. The corresponding 100 Rayleigh traces of this event, recorded when $N=1$, are shown in Fig. 3(d). As shown in Figs. 3(e) and 3(f), respectively, the vibrations position and frequency of this event can be also identified efficiently.
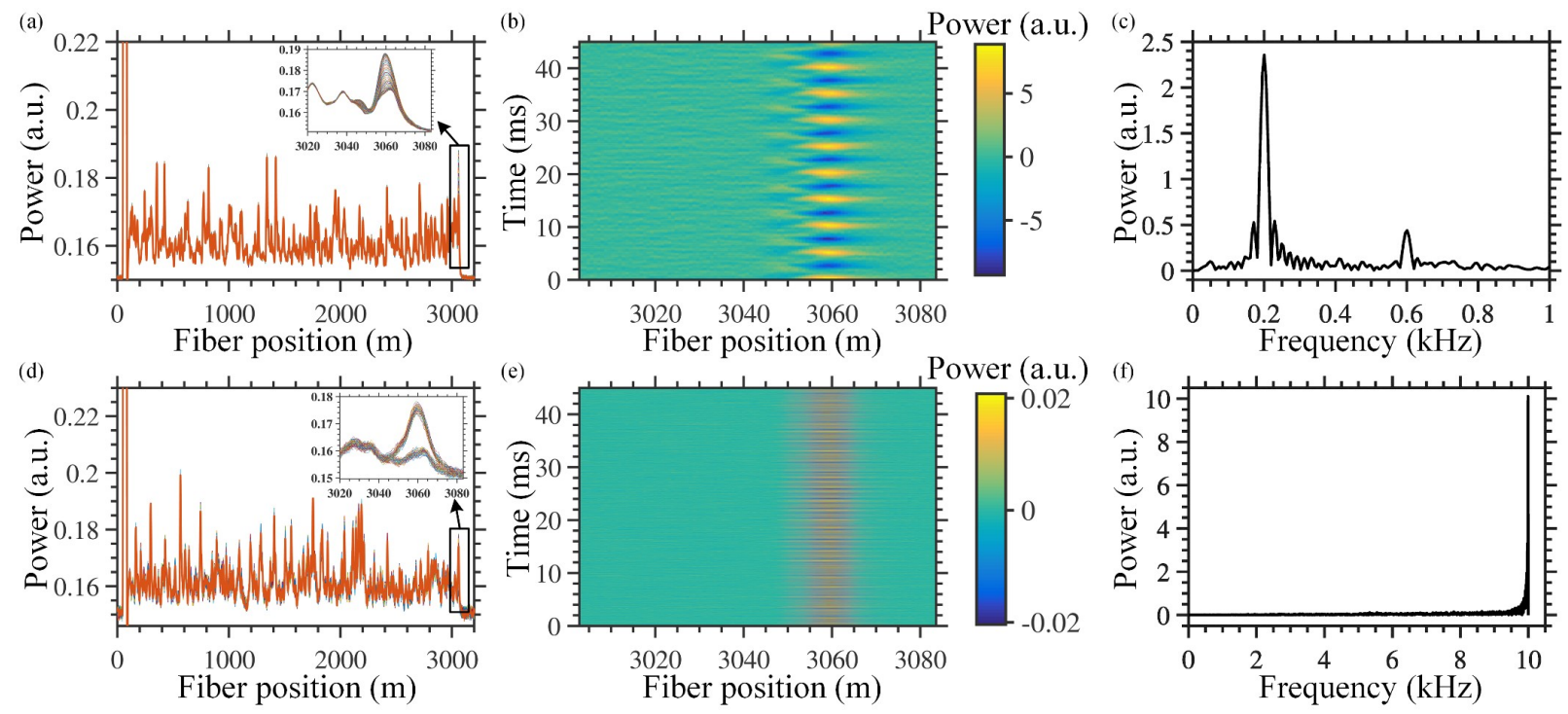

Figure. 3. Sensing of PZT vibrations with $200 \mathrm{~Hz}$ frequency: (a) 100 consecutive averaged Rayleigh traces. The inset shows a zoomin image around the PZT location. (b) Position information and (c) power spectrum of the $200 \mathrm{~Hz}$ vibrations event. Sensing of PZT vibrations with $10 \mathrm{kHz}$ frequency: (d) 100 consecutive raw Rayleigh traces. The inset shows a zoom-in image around the PZT location. (e) Position information and (f) power spectrum of the $10 \mathrm{kHz}$ vibrations event.

\section{COMPARISON BETWEEN SMF- AND FMF-BASED DAS}

In the forward pump direction, we show that aligning the axes of the SMF and TMF followed by joining them is necessary for the QSM operation within the TMF. In the backward direction, however, Rayleigh scattering excites the entire modes supported by the fiber, even if the pump signal includes only the fundamental mode $\left[{ }^{15}\right]$. Although, in the QSM operation, the power of the backscattered Rayleigh signal is dominated by that of the $\mathrm{LP}_{01}$ mode $\left[{ }^{15}\right]$, recording the signals of whole modes would introduce noise into the DAS system. Therefore, the SMF-TMF connection in our design has another major advantage, rather than supporting the QSM operation in the pump direction within the TMF, which is the SMF acts as a spatial filter in the backward direction and only collects the Rayleigh signal of the fundamental mode. In other words, our design behaves similarly as the SMF based DAS with the exception of the opportunity of pumping pulses with higher peak-powers because of the larger effective area of the TMF, in comparison with the SMF. To compare the performances of the standard SMF (Corning, SMF-28) and TMF based DAS, we separately use the two fibers in the experimental setup shown in Fig. 2 when using the PZT cylinder as the vibration source. In the two separate 
experiments, we fix all of the experimental parameters including the pulse width and peak-power, fiber length attached to the PZT, and vibrations frequency and amplitude of the PZT. Figures 4(a) and 4(b) respectively show the power spectra of the PZT vibrations events sensed by the SMF and TMF, when the PZT is vibrating with a $200 \mathrm{~Hz}$ frequency. We define the SNR as the ratio between the peak-power in the spectrum and the mean noise floor power. We find that the SNR values when using the SMF and TMF are $14.9 \mathrm{~dB}$ and $15.6 \mathrm{~dB}$, respectively. This result indicates that the TMF has comparable performance with the SMF when detecting the fundamental vibrations frequency. However, the SMF based DAS has more power distributed among the high-order harmonic frequencies.
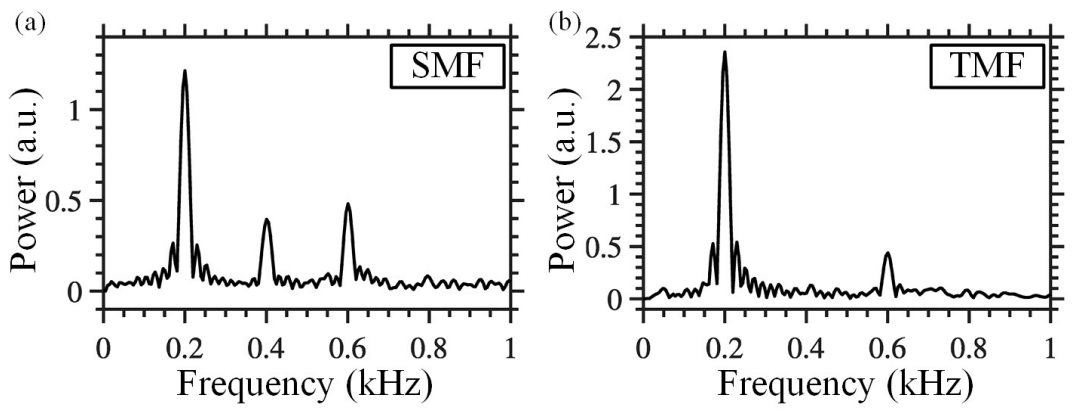

Figure 4. Power spectrum of the $200 \mathrm{~Hz}$ PZT vibrations event when sensed using (a) SMF and (b) TMF.

\section{CONCLUSION}

In summary, we experimentally demonstrate the possibility of designing an $\Phi$-OTDR based DAS using a QSM operated TMF. The designed system can successfully detect low (e.g., $200 \mathrm{~Hz}$ ) and high (the Nyquist frequency of our experimental setup) vibrations frequencies produced by a PZT cylinder. The designed FMF-based DAS has a comparable SNR to that of the standard SMF-based DAS. Our design paves the way towards designing a long-range and high spatial resolution DAS that could outperform the typical standard SMF based DAS.

\section{ACKNOWLEDGEMENTS}

The authors gratefully acknowledge the financial support provided to this work by the Saudi Aramco under Grant No. RGC/3/3138-01. This work is partially supported by King Abdullah University of Science and Technology (KAUST) baseline funding (BAS/1/1614-01-01), KAUST equipment funding KCR/1/2081-01-01, and GEN/1/6607-01-01.

\section{REFERENCES}

[1] Ryf, R., Randel, S., Gnauck, A. H., Bolle, C., Sierra, A., Mumtaz, S., Esmaeelpour, M., Burrows, E. C., Essiambre, R. J., Winzer, P. J., Peckham, D. W., McCurdy, A. H. and Lingle, R., "Mode-division multiplexing over $96 \mathrm{~km}$ of few-mode fiber using coherent 6×6 MIMO processing," J. Light. Technol. 30(4), 521-531 (2012).

[2] Li, A., Wang, Y. and Shieh, W., "Few-mode fiber based optical sensors," 2015 IEEE Photonics Conf. IPC 2015 23(2), 319-320 (2015).

[3] Lim, J., Yang, Q., Jones, B. E. and Jackson, P. R., "Strain and temperature sensors using multimode optical fiber bragg gratings and correlation signal processing," IEEE Trans. Instrum. Meas. 51(4), 622-627 (2002).

[4] Sun, A. and $\mathrm{Wu}, \mathrm{Z}$., "Multimode interference in single mode-multimode FBG for simultaneous measurement of strain and bending," IEEE Sens. J. 15(6), 3390-3394 (2015).

[5] Ashry, I., Wang, A. and Xu, Y., "Mode-division-multiplexing of absorption-based fiber optical sensors," Opt. Express 24(5), 5186 (2016).

[6] Ashry, I., Wang, A. and Xu, Y., "Mode-Based Reconstruction of Chemical Distributions in Optical Fibers," IEEE J. Sel. Top. Quantum Electron. 23(2) (2017).

[7] Weng, Y., Ip, E., Pan, Z. and Wang, T., "Single-end simultaneous temperature and strain sensing techniques based on Brillouin optical time domain reflectometry in few-mode fibers," Opt. Express 23(7), 9024 (2015).

[8] Wang, M., Wu, H., Tang, M., Zhao, Z., Dang, Y., Zhao, C., Liao, R., Chen, W., Fu, S., Yang, C., Tong, W., Shum, P. P. and Liu, D., "Few-mode fiber based Raman distributed temperature sensing," Opt. Express 25(5), 4907 (2017).

[9] Yuan Mao, Islam Ashry, Mohd Sharizal Alias, Tien Khee Ng, Frode Hveding, Muhammad Arsalan, and Boon S. 
Ooi, "Investigating the performance of a few-mode fiber for distributed acoustic sensing," IEEE Photonics J., DOI: 10.1109/JPHOT.2019.2940951 (2019).

[10] Chen, M., Masoudi, A., Parmigiani, F. and Brambilla, G., "Distributed acoustic sensor based on a two-mode fiber," Opt. Express 26(19), 25399 (2018).

[11] Bao, X., Zhou, D. P., Baker, C. and Chen, L., "Recent Development in the Distributed Fiber Optic Acoustic and Ultrasonic Detection,” J. Light. Technol. 35(16), 3256-3267 (2017).

[12] Lu, P., Ashry, I. and Xu, Y., "Adaptive Mode Control for Few-Mode Fiber Based Sensors and Sensor Networks," J. Light. Technol. (2017).

[13] Ashry, I., Mao, Y., Alias, M. S., Ng, T. K., Hveding, F., Arsalan, M. and Ooi, B. S., "Normalized differential method for improving the signal-to-noise ratio of a distributed acoustic sensor," Appl. Opt. 58(18), 4933 (2019).

[14] Ren, M., Zhou, D.-P., Chen, L. and Bao, X., "Influence of finite extinction ratio on performance of phase-sensitive optical time-domain reflectometry," Opt. Express 24(12), 13325 (2016).

[15] Wang, Z., Wu, H., Hu, X., Zhao, N., Mo, Q. and Li, G., "Rayleigh scattering in few-mode optical fibers," Sci. Rep. 6 (2016). 